\author{
二次电池用局部高浓度电解质的研究进展与展望

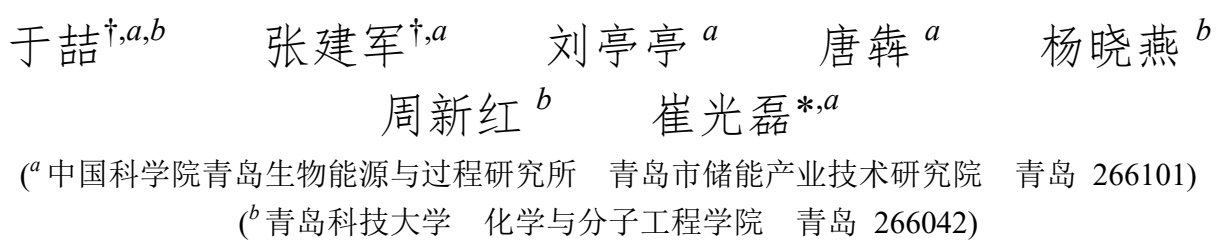

\begin{abstract}
摘要 电解质作为二次电池离子传导的重要介质, 对于提升二次电池循环稳定性能、安全性能等方面起着至关重要的 作用. 局部高浓度电解质是指在高浓度电解质中加入 “稀释剂”, 形成盐的局部高浓度状态, 既能兼具高浓度电解质的 优异特性，又具有低成本和优良润湿性的特点，应用前景非常广阔. 近几年，局部高浓度电解质在阻燃锂金属电池、高 电压锂电池、低温锂电池、锂硫电池和钠电池等多方面应用广泛, 且展现出非常好的使用效果. 本综述重点从局部高 浓度电解质的功能性应用角度出发，详细阐述了局部高浓度电解质的类型、制备、作用机理及其在不同二次电池中的 功能性应用进展和主要研究现状, 文末还对局部高浓度电解质的未来可能发展趋势进行了分析与展望.
\end{abstract}

关键词 局部高浓度电解质; 二次电池; 功能性应用

\title{
Research Progress and Perspectives of Localized High-concentration Electrolytes for Secondary Batteries
}

\author{
Yu, Zhe $\mathrm{e}^{\dagger, a, b}$ \\ Zhang, Jianjun ${ }^{\dagger, a}$ \\ Liu, Tingting ${ }^{a}$ \\ Tang, Ben ${ }^{a}$ \\ Yang, Xiaoyan ${ }^{b}$ \\ Zhou, Xinhong ${ }^{b}$ \\ Cui, Guanglei*,a \\ ( ${ }^{a}$ Qingdao Institute of Bioenergy and Bioprocess Technology, Chinese Academy of Sciences, Qingdao Industrial Energy \\ Storage Technology Institute, Qingdao 266101, China) \\ $\left({ }^{b}\right.$ College of Chemistry and Molecular Engineering, Qingdao University of Science \& Technology, Qingdao 266042, China)
}

\begin{abstract}
The electrolyte, which is an important medium of ion conduction for secondary batteries, plays a crucial role in improving the cycling performance and safety performance of secondary batteries. Localized high-concentration electrolytes, which are formed by adding "diluent" into high-concentration electrolyte, not only reserve the outstanding properties of high-concentration electrolytes but also possess low viscosity, excellent wettability and low cost, promising broad application prospect. Localized high-concentration electrolytes have already played a part in flame-retardant lithium battery, high-voltage lithium battery, low-temperature lithium battery, lithium sulfur battery and sodium battery. Herein, this paper mainly reviews the types and preparation of localized high-concentration electrolytes and their functional mechanism and research status in various secondary batteries. We discuss the challenges and future development of localized high-concentration electrolytes and have the outlook at the end of the paper.
\end{abstract}

Keywords localized high-concentration electrolyte; secondary battery; functional application

\section{1 引言}

二次锂电池是当前应用最为广泛的二次清洁电化 学储能器件 ${ }^{[1 \sim 7]}$. 近几年, 锂离子电池(LIB)技术已经大 范围应用于手机、笔记本等便携式电子设备等领域, 且 正在向动力电池和大规模储能等领域进军. 如何兼顾高 能量密度和高安全性能, 是目前国内外研究者关注的重 点 $^{[8 \sim 11]}$.

电解质是连接正负极的重要载体, 且其与正负极所 形成的界面关系到电池的能量密度、循环寿命和安全性
能 ${ }^{[12 ~ 14]}$. 传统的锂离子电池电解液为 $1 \mathrm{~mol} / \mathrm{L}$ 的六氟磷 酸锂 $\left(\mathrm{LiPF}_{6}\right.$ )/碳酸亚乙酯(EC)/线性碳酸酯(如碳酸二甲 酯). EC 的还原分解产物可在石墨表面形成稳定的固态 电解质界面(SEI); $\mathrm{LiPF}_{6}$ 可以在正极集流体铝箔上形成 $\mathrm{AlF}_{3}$ 膜, 有效抑制电解液对铝集流体腐蚀. 然而, 当有 微量水存在或者处于较高温度时, $\mathrm{LiPF}_{6}$ 会发生严重的 分解, 即使存在添加剂, $\mathrm{LiPF}_{6}$ 的分解也不会被抑制, 导 致电解质的电化学窗口也偏低, 限制了其在高质量能量 密度和高温二次锂电池中的应用, 因此, 迫切需要开发 新型的高安全电解质体系. 目前, 科研工作者主要聚焦

* E-mail: cuigl@qibebt.ac.cn; Tel.: 0532-80662746; Fax: 0532-80662744

$\uparrow$ These authors contributed equally to this work.

Received October 25, 2019; published January 20, 2020.

Project supported by the National Natural Science Foundation of China (Nos. 51703236, 51625204).

项目受国家自然科学基金(Nos. 51703236, 51625204)资助. 
于离子液体、聚合物固态电解质、无机固态电解质和高 浓度电解质等方面. 在这其中, 高浓度电解质由于具有 多方面优异特性而格外受到关注 ${ }^{[15 ~ 20]}$.

所谓高浓度电解质是指随着盐浓度的不断增加, 盐 的阳离子和阴离子与溶剂之间的相互作用增强, 自由态 溶剂分子的含量大大减少. 在非水溶剂当中, 当盐的浓 度 $\geqslant 3.5 \mathrm{~mol} / \mathrm{L}$ 时 ${ }^{[21 ~ 24]}$, 自由溶剂分子基本消失, 形成一 种具有特殊结构的新型电解质, 称为高浓度电解质. 高 浓度电解质与传统的低浓度 (如 $1 \mathrm{~mol} / \mathrm{L}$ ) 电解质性质完 全不同，且具有如下特殊优良特性: 1. 高离子迁移数; 2 . 防止铝集流体腐蚀; 3. 有效抑制锂枝晶: (1)在锂电池充 放电循环过程中高浓度锂盐电解质能够提供足够的锂 离子, 形成均匀的离子流, 均衡电场, 进而有效抑制锂 枝晶的产生; (2)高浓度电解质会形成独特的 SEI. 与传 统低浓度电解质形成的 SEI(主要由碳酸酯类溶剂分解 产生)相比, 高浓度电解质的 SEI 来源于电解质中盐的 阴离子(FSI ${ }^{-}, \mathrm{TFSI}^{-}$等)还原分解, 易生成 $\mathrm{LiF}$, 而富含 LiF 的 SEI 相对稳定, 从而可以有效减少界面副反应, 达到抑制锂枝晶的目的 ${ }^{[25 ~ 29]} ; 4$. 拓宽电化学窗口: (1)电 解液中自由溶剂分子大大减少, 从而从根本上减少了不 稳定溶剂与正极的反应; (2)高浓度电解质独特的 3D 溶 液结构, 很大程度上阻碍了正极过渡金属离子溶出, 对 负极也会产生积极影响; 5. 提升阻燃性能, 安全性提高: 锂离子电池由高活性的正极材料和有机电解液组成, 在 受热条件下易发生 “热失控”, 引发危险事故, 而高浓 度电解质中强烈的溶剂化作用, 自由溶剂分子减少, 抑 制了易燃溶剂的挥发, 阻燃性能得以提升 ${ }^{[30,31]}$.

综上所述, 与传统 $1 \mathrm{~mol} / \mathrm{L}$ 稀溶液电解液体系相比, 高浓度电解质有着显著的优势. 然而, 高浓度电解质的 大规模商业化推广应用仍然面临着巨大挑战, 具体表现 为: (1)成本较高. 锂盐(主要为 $\mathrm{LiPF}_{6}$ ) 在商业化锂离子电 池液态电解液成本中占有超过 $70 \%$ 的比例, 因此电解液 中锂盐浓度增加必将极大提高成本; 更为重要的是, 随 着锂离子电池行业要求的提升, 一些新型锂盐显示出良 好的发展潜力, 目前市场上已开始少量应用的新型电解 质锂盐包括双草酸硼酸锂 (LiBOB)、二氟草酸砋酸锂 (LiODFB)、双(氟磺酰)亚胺锂(LiFSI)、双(三氟甲烷磺酰) 亚胺锂(LiTFSI)等, 然而, 由于这些新型锂盐生产成本 更高, 这进一步提升了高浓度电解质的成本; (2)高粘度. 高浓度电解质中的盐含量增加会引起粘度的增加; (3)对 电极或隔膜浸润性差, 造成对正负极极片和隔膜浸润性 的明显降低, 最终后果是二次电池在循环过程中会出现 不同程度的 “干区”, 造成电池阻抗的持续增加进而大 大缩短循环寿命.

为解决高浓度电解质存在的上述瓶颈问题, 近年来 提出了局部高浓度电解质新概念. 局部高浓电解质的维 形早已有之, 但是这个名称是由张继光等 ${ }^{[32]}$ 在近几年 高度概括后提出的.
通过向高浓度电解质(HCE)中添加一种 “惰性” 稀 释剂, 形成局部高浓度电解质(LHCE). 稀释剂本身意味 着与高浓度电解质具有相似或更宽的电化学窗口，其关 键条件为不能溶解盐, 但与溶剂可以互溶, 形成 $\left(\mathrm{Li}^{+}-\right.$ 溶剂)稀释剂)状态. 它不会影响高浓度电解质中原有的 盐一溶剂的配位, 但能显著降低单位体积中盐的含量, 从而降低盐的成本, 同时保留(甚至增强)HCE 的独特性 能，更符合实际应用. 在分子水平上理解，局部高浓度 电解质可以看作是高浓度电解质与稀释剂的混合物(图 1). 虽然 HCE 和稀释剂均与正负极接触, 但电解质中载 流子 $\left(\mathrm{Li}^{+} 、 \mathrm{Na}^{+}\right.$等)优先在 HCE 区域内移动. 基于这一原 理, 通过仔细选择适用的锂盐、溶剂和稀释剂, 实验调 试三者的比例，就能实现 “低浓度、低成本” 的高浓度 电解质在二次电池中应用, 称之为 “局部高浓度电解 质”。

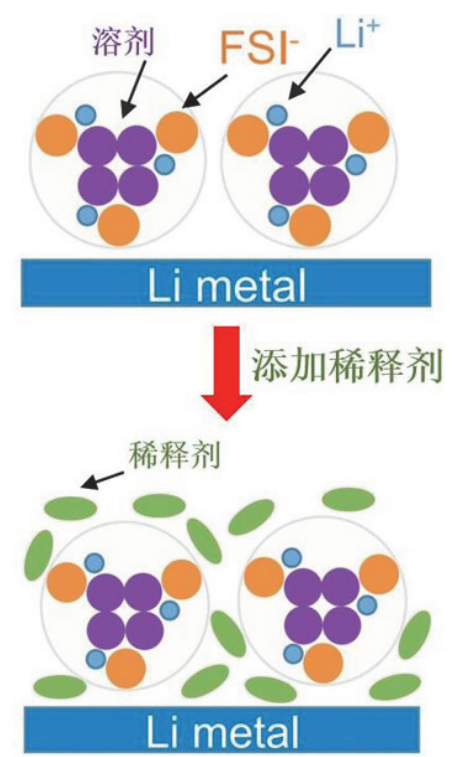

图 1 局部高浓度电解质体系结构示意图(以 $1.2 \mathrm{~mol} / \mathrm{L} \mathrm{LiFSI} /$ 双(2,2,2三氟乙基)醚(BTFE)为例) ${ }^{[32]}$

Figure 1 The schematical images of localized high-concentration electrolytes (Take $1.2 \mathrm{~mol} / \mathrm{L} \mathrm{LiFSI} / \mathrm{TEP}-\mathrm{BTFE}$ for example)

所谓局部高浓度电解质是指在高浓度电解质中添 加与溶剂能够互溶, 但与盐不互溶的稀释剂, 稀释剂加 入后, 不影响原高浓度电解质中盐-溶剂的特殊配位结 构, 从而形成盐的局部高浓度状态. 这样既保证了高浓 度电解质的特性, 又具有自己独有的特性 ${ }^{[33]}$. 其黏度远 低于高浓度电解质，且对极片和隔膜均具有优异的润湿 性. 用于局部高浓电解质的稀释剂需要满足以下特征: (1)低介电常数和低络合能力, 能与溶剂相容, 且不影响 原高浓度电解质中盐-溶剂的特殊配位结构; (2)低粘度 和高润湿性; (3)成本低; (4)阻燃; (5)有利于形成良好的 SEI. 2,2,2-三氟乙基-1,1,2,2-四氟乙基醚(HFE)、1,1,2,2四氟乙基-2,2,3,3,-四氟丙基醚(TTE)等溶剂符合了以上 特征(图 2). 

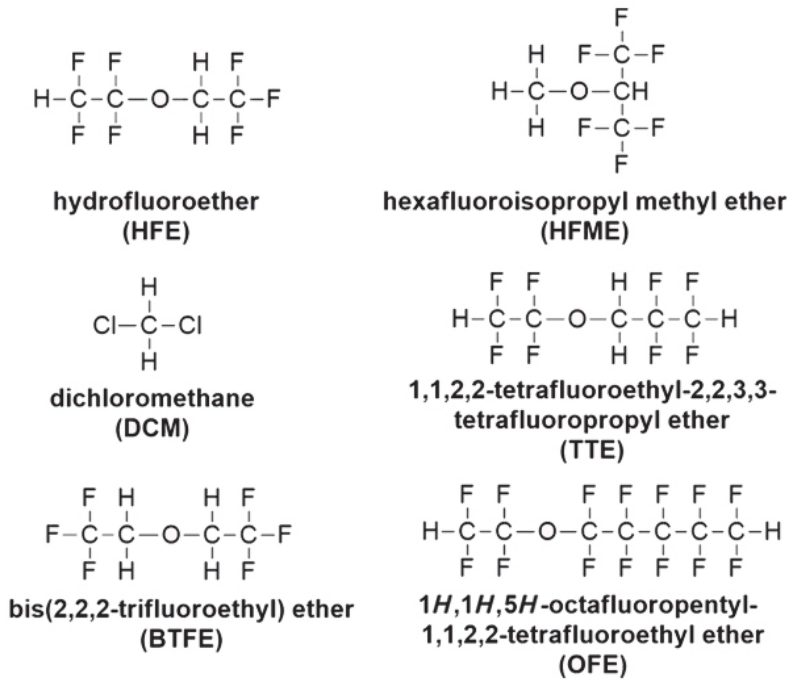

图 2 稀释剂分子结构式

Figure 2 Molecular structures of diluent

目前采用的氟代醚类稀释剂与烷类稀释剂通常具 有低极性、低介电常数和低粘度的特点, 无法溶解盐. 氟代醚由于具有吸电子的氟原子而具有较高的抗氧化稳 定性, 适合作为高浓盐电解质体系的稀释剂, 其中 HFE 为最常用的稀释剂, HFE 分子的最高占据分子轨道 (HOMO) 能级 $(-13.91 \mathrm{eV})$ 低于碳酸丙烯酯(PC) 分子的 HOMO 能级 $(-12.55 \mathrm{eV})$, 说明 HFE 的抗氧化稳定性高 于 PC. 将纯三乙二醇二甲醚 $(\mathrm{G} 3)$ 和纯 HFE 相比, HFE 具 有较低的粘度, 在 $30{ }^{\circ} \mathrm{C}$ 时的粘度分别为 $1.89 \mathrm{mPa} \cdot \mathrm{s}$ 和 $1.30 \mathrm{mPa} \cdot \mathrm{s}$. 在室温 $25{ }^{\circ} \mathrm{C}$ 时, HFE 的相对介电常数 $\left(\varepsilon_{\mathrm{r}}=\right.$ $6.21)$ 相对低于其它常见的溶剂, 如 PC $\left(\varepsilon_{\mathrm{r}}=64.9\right) 、 1,3-$ 二氧环戊烷 $(\mathrm{DOL})\left(\varepsilon_{\mathrm{r}}=7.2\right) 、 \mathrm{G} 3\left(\varepsilon_{\mathrm{r}}=7.63\right)$ 和水 $\left(\varepsilon_{\mathrm{r}}=78.4\right)$, 稀释剂 BTFE 的介电常数 $\left(\varepsilon_{\mathrm{r}}=4.33\right)$ 更低. HFE 的 DN 值 (Gutman's donor number)约为 10 , 低于 G3 (14.0)等溶剂. 因此, 得出结论, HFE 几乎不能溶解盐, 并且电解质中 其它溶剂分子优先与阳离子溶剂化, 若将 HFE 应用在 锂硫电池中, 还可起到抑制多硫化物溶解的作用 ${ }^{[59]}$. 将 1,1,1,3,3,3-六氟异丙基甲基醚(HFME)作为稀释剂时, LiTFSI 在其中不能溶解, 然而, HFME 在常用的碳酸酯 类溶剂中可以混溶, 并且具有较宽的电化学窗口. 此外, HFME 具有较低的粘度和较好的润湿性. 这些化学和物 理性质表明, HFME 是实现 “拟高浓度电解质” 的理想 辅助溶剂。 $1 H, 1 H, 5 H$-八氟戊基-1,1,2,2-四氟乙基醚 (OFE) 作为稀释剂时, 由于本身具有较低的蒸汽压 (989.3 Pa), 较高的沸点 $\left(133{ }^{\circ} \mathrm{C}\right)$ 和较高的闪点 $\left(45.037{ }^{\circ} \mathrm{C}\right)$, 不会发生燃烧现象, OFE 可作为阻燃稀释 剂, 应用于局部高浓度电解质当中, 提升阻燃性能.

最近, 局部高浓度电解质已经成功应用于阻燃二 次锂电池、高电压二次锂电池、低温二次锂电池、锂硫 电池和钠电池等多种电化学储能器件中, 且效果显著.
因此本综述通过全方位调研前期文献资料重点论述了 目前国内外局部高浓度电解质的研究进展以及前沿关 键科学问题, 并且从功能性应用进展角度对该新型电 解质体系进行系统分析和梳理, 最后对其作为二次电 池关键电解质材料所面临的主要挑战和应用前景进行 了展望.

\section{2 局部高浓度电解质在阻燃二次电池中的应用}

安全隐患已严重制约了锂离子电池的广泛使用, 因此高安全阻燃二次电池的开发至关重要. 商品化锂 离子电池中通常采用碳酸酯基液态电解液, 其主要由 锂盐和碳酸酯溶剂组成. 锂盐主要为 $\mathrm{LiPF}_{6}$; 碳酸酯溶 剂主要包括 EC、PC、碳酸二甲酯(DMC)、碳酸二乙酯 (DEC) 和碳酸甲乙酯(EMC). 碳酸酯基电解液闪点低, 易挥发、高度易燃, 因此采用该电解液的二次锂电池起 火爆炸事故层出不穷. 开发二次锂电池用阻燃电解液刻 不容缓!

为实现电解液阻燃, 通常是在常规液态电解液中加 入阻燃剂, 但阻燃剂加入后通常不能形成稳定的 SEI, 导致电池性能大打折扣 ${ }^{[34]}$. 离子液体和固态电解质, 虽 然也能提升二次电池安全性能, 但离子液体的高粘度高 成本、聚合物固态电解质的低室温离子电导率以及无机 固态电解质过高的电极/电解质固/固接触阻抗, 都在很 大程度上严重影响和制约了它们的广泛应 用 ${ }^{[35 \sim 40]}$.

为进一步开发新型阻燃电解质, 2018 年, 张继光 等 ${ }^{[32]}$ 报道了一种局部高浓度阻燃电解质, 将阻燃剂 TEP 和 BTFE 以物质的量比 $1: 3$ 的比例混合, 然后加入锂 盐, 最后得到 $1.2 \mathrm{~mol} / \mathrm{L} \mathrm{LiFSI}$ 局部高浓度阻燃电解质. 实验结果表明: 该局部高浓度阻燃电解质, 阻燃性能优 异; 同时兼顾了低浓度电解液低粘度、低造价的优势. 采用这种电解质组装的三元正极材料 $\left(\mathrm{LiNi}_{0.6} \mathrm{Mn}_{0.2}-\right.$ $\left.\mathrm{Co}_{0.2} \mathrm{O}_{2}\right) / \mathrm{Li}$ 金属电池, 在 $1 \mathrm{C}\left(1.6 \mathrm{~mA} / \mathrm{cm}^{2}\right)$ 下循环 600 次 后, 容量保持率超过 $97 \%$ (图 3). 性能优异的原因主要为 $1.2 \mathrm{~mol} / \mathrm{L} \mathrm{LiFSI} / \mathrm{TEP}-\mathrm{BTFE}$ 电解质在锂金属电池中能够 形成 $\mathrm{Li}^{+}-\mathrm{FSI}^{-}-\mathrm{TEP}$ 的溶剂化结构, 提升了界面反应动力 学, 构筑了优异的界面, 有效稳定锂负极和抑制锂枝晶 生成. 该局部高浓度阻燃电解质为解决高能量密度二次 锂电池的安全性问题提供了非常好的解决方案, 并且对 未来的相关研究工作提供了非常好的思路借鉴和理论 指导.

2019 年, 王春生课题组 ${ }^{[41]}$ 制备出一种新型的局部 高浓度阻燃电解质, 应用于锂硫电池中, 且获得了较好 的电化学性能. 作者将乙二醇二甲醚(DME)和 OFE 按照 体积比(50：50;15：85;5：95)混合后, 加入 LiFSI 制成 $1 \mathrm{~mol} / \mathrm{L}$ 的溶液, 发现随着 OFE 含量的增加且 OFE 与 DME 体积比达到 $95: 5$ 时, 电解质具有阻燃性能, 且组 装的 $\mathrm{Li} / \mathrm{Li}$ 对称电池, $\mathrm{Li}$ 沉积/剥离的库伦效率高达 $99.3 \%$, 使用扫描电镜发现: 锂片上的沉积更加规则且 


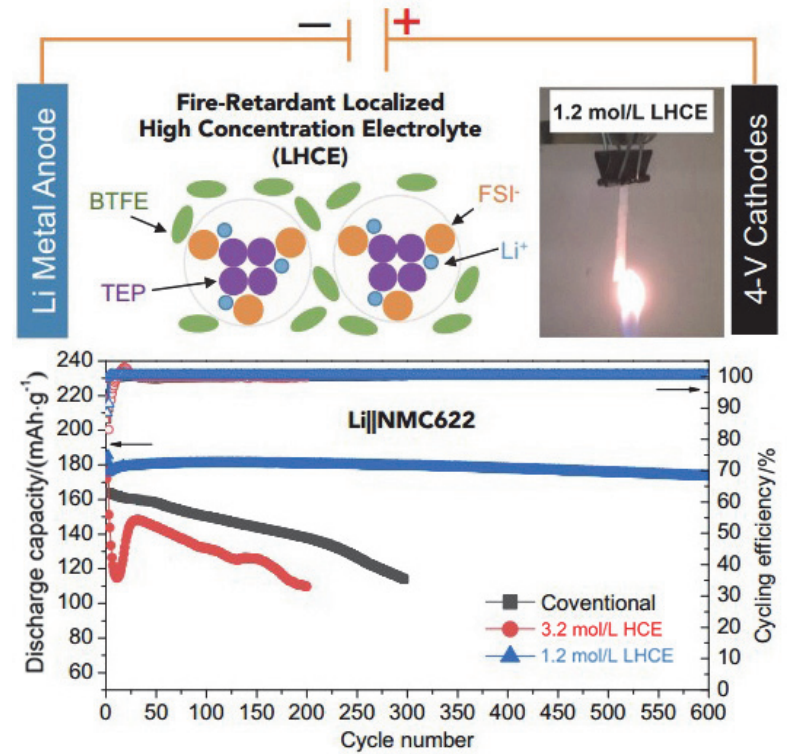

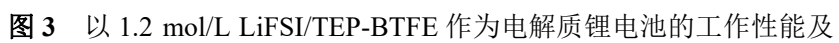
原理图

Figure 3 The working principle and performance of $1.2 \mathrm{~mol} / \mathrm{L}$ $\mathrm{LiFSI/TEP-BTFE} \mathrm{electrolyte} \mathrm{for} \mathrm{lithium} \mathrm{ion} \mathrm{battery}$

均匀. 这表明该局部高浓度电解质能够起到较好的抑制 锂枝晶的作用. 为深入探究该电解质有效抑制锂枝晶的 内在原因, 作者分别使用 $V(\mathrm{OFE}): V(\mathrm{DME})=95: 5$ (DME5)和 $V(\mathrm{OFE}): V(\mathrm{DME})=50: 50$ (DME50) 电解质 组装锂金属电池. 循环 50 周后, 拆解出锂金属负极进行 $X$ 射线光电子能谱(XPS)测试, 研究结果表明: 随着 OFE 含量的升高, 锂金属表面的 $\mathrm{Li}-\mathrm{F}$ 键也逐渐增多, 形成了富氟的 SEI, 可以有效地抑制锂枝晶的形成. 与 此同时还发现该局部高浓度阻燃电解质能够抑制长链 多硫化物的溶解, 抑制 “穿梭效应”, 赋予锂硫电池较 好的电化学性能: 基于碳硫正极的锂硫电池在 100 $\mathrm{mA} / \mathrm{g}$ 的电流密度下循环 150 周后仍有 $775 \mathrm{mAh} / \mathrm{g}$ 的放 电比容量. 此项工作为开发锂硫电池用新型局部高浓度 阻燃电解质体系打下了一个非常好的开端, 但其是否能 用于高面密度的纯硫正极体系仍需要进一步的研究和 验证.

许武、张继光和贾海平等 ${ }^{[42]}$ 将阻燃局部高浓度电解 质, 应用于高性能硅电池当中. 使用 BTFE 作为添加剂, 在阻燃剂 TEP 中添加质量分数为 $w=1.2 \%$ 的氟代碳酸 乙烯酯(FEC)得到阻燃局部高浓度电解质 $(1.2 \mathrm{~mol} / \mathrm{L}$ LiFSI/(TEP-FEC)-BTFE), 并将其应用于硅碳负极材料 $(\mathrm{Si} / \mathrm{Gr})$ 电池当中. 通过自熄时间测试(SETs)发现局部高 浓度电解质仍具有优异的阻燃性能, 原因为加入稀释剂 BTFE 后, 电解质体系中的 TEP 含量仍能够充分起到阻 燃作用. 含少量 FEC 的 LHCE 能显著增强正极和负极的 表面化学性质, 抑制正极在嵌锂状态下的膨胀, 具有良 好的电化学性能和良好的长循环稳定性和高温性能. 在 电解质中形成局部高度配位的 $\mathrm{Li}^{+}-\mathrm{TEP}$ 结构, 并且在硅 负极形成由盐阴离子分解生成的富 LiF 的 SEI. 因此
$\mathrm{Si} / \mathrm{Gr} / \mathrm{Li}$ 半电池和 $\mathrm{NMC} / \mathrm{Si} / \mathrm{Gr}$ 全电池均表现出优异的循 环稳定性, 使用含有 $w=1.2 \% \mathrm{FEC}$ 的局部高浓电解质 的 $\mathrm{Si} / \mathrm{Gr} / \mathrm{Li}$ 半电池在 300 次时仍然具有 $73.4 \%$ 的容量保 持率和 $>99 \%$ 的库伦效率. 此工作的发现为硅基高能量 密度锂电池 ${ }^{[43]}$ 开发开辟了新的途径.

局部高浓度电解质在阻燃二次电池中的应用方面, 仍然有一些问题需要进一步思考与完善:

(1)目前局部高浓度阻燃电解质的种类比较单一, 不够丰富. 比如溶剂体系仅仅局限于 TEP; 共溶剂仅仅 局限于氟代醚. 因此是否可以探究和发现更多局部高浓 度阻燃电解质的种类 ${ }^{[38]}$ ? 使用更多的(卤代)磷酸酯类阻 燃溶剂、(卤代)亚磷酸酯类阻燃溶剂、(卤代)膦酸酯类 阻燃溶剂、磷腈类等含磷元素的有机阻燃溶剂, 如磷酸 三甲酯(TMP)、磷酸三苯酯(TPP)、磷酸三丁酯(TBP)、

二甲基甲基膦酸酯(DMMP)等. (2)能否开发新型低黏度 局部高浓度离子液体基阻燃电解质? (3)能否开发性能 优异的阻燃型凝胶聚合物电解质? (4)局部高浓度阻燃 电解质应用于大容量的软包电池中是否会出现漏液、产 气等问题? 这需要在后续局部高浓度阻燃电解质应用 于大容量软包电池制备过程时，做进一步的考察、思考、 改进以及完善.

\section{3 局部高浓度电解质在高电压二次锂电池中的 应用}

随着便携式电子设备、电动汽车、大规模电网的快 速发展, 人们迫切需求开发出高能量密度二次锂电池. 科研人员不停地探索具有较大电势差的正极和负极, 以 最大限度地提高电池的工作电压并且得到更高的比容 量. 在正极方面: 具有高压和大容量优点的正极材料, 引起了人们的广泛关注; 负极方面: 锂金属电池, 由于 采用质量很轻的金属锂, 具有远高于传统锂离子电池的 能量密度. 然而, 金属锂高度活泼, 极易与电解液发生 反应，并且伴有锂枝晶的产生，从而导致锂金属电池的 循环寿命有限、且存在很大的安全隐患, 无法达到实用 要求. 为实现高电压锂金属二次电池的应用, 张继光课 题组陆续展开了一系列研究工作.

2017 年, 张继光等 ${ }^{[4]}$ 发表了一篇关于局部高浓度 电解质用于高电压锂金属电池的研究论文, 该工作开发 了一种新型的 “局部高浓度电解液” : $1.2 \mathrm{~mol} / \mathrm{L} \mathrm{LiFSI}$ 溶于 DMC/BTFE 混合溶剂(溶剂物质的量比为 $1: 2$ ). 通 过向高浓度电解质中加入惰性稀释剂 BTFE, 稀释剂本 身络合能力很低不能溶解锂盐, 但能与 DMC 互溶, 因 此它没有影响 HCE 中原有的锂盐与溶剂的配位, $\mathrm{Li}^{+}$会 优先在 HCE 内迁移, LHCE 在保持(甚至增强)HCE 独特 性能的同时，显著降低了 $\mathrm{Li}$ 盐的总浓度，使电解质达到 更实用的水平. LHCE 在保持高浓度电解质稳定电极/电 解液界面的同时，还可以匹配高电压正极材料，推动了 金属锂的实用化进程. 电化学数据表明: $1.2 \mathrm{~mol} / \mathrm{L}$ 
LiFSI/DMC-BTFE 局部高浓度电解液能够匹配高电压正 极 $\mathrm{LiNi}_{1 / 3} \mathrm{Mn}_{1 / 3} \mathrm{Co}_{1 / 3} \mathrm{O}_{2}$, 并且在 $\mathrm{Li} / \mathrm{Cu}$ 电池测试中有较高 的库伦效率 $(99.2 \%)$, 且无锂枝晶产生, 电池表现出良好 循环稳定性. 当使用 $1.0 \mathrm{~mA} / \mathrm{cm}^{2}$ 和 $3.0 \mathrm{~mA} / \mathrm{cm}^{2}$ 的电流 密度时, 具有较高的库伦效率, 分别达到 $99.4 \%$ 和 $98.9 \%$. 电流密度为 $3.0 \mathrm{~mA} / \mathrm{cm}^{2}$ 相当于实际面容量为 3.0 $\mathrm{mAh} / \mathrm{cm}^{2}$ 的电池 $1 \mathrm{C}$ 的速率(对于大多数应用来说已经 足够). 即使在电流密度为 $5.0 \mathrm{~mA} / \mathrm{cm}^{2}$ 的情况下, 过电 位也只有 $120 \mathrm{mV}$, 说明 LHCE 中具有良好的反应动力 学. 通过扫描电镜(SEM)观察了不同电解质中 $\mathrm{Li}$ 沉积形 态的显著差异. 在常规电解质中观察到高度多孔疏松的 结构, 并有树枝状锂生长(这与数据中较低的库伦效率 一致). 当使用 $1.2 \mathrm{~mol} / \mathrm{L} \mathrm{LiFSI/DMC-BTFE}[n(\mathrm{DMC})$ ： $n(\mathrm{BTFE})=1: 2]$ 时, 形成了致密的无枝晶的锂颗粒聚集 体, 并且 LHCE 中沉积的 $\mathrm{Li}$ 层厚度仅为 $10 \mu \mathrm{m}$, 这个值 代表了电化学沉积得到的最致密的 $\mathrm{Li}$ 沉积层之一. 形 成高密度、低表面积、粒径较大的节点状 $\mathrm{Li}$ 沉积, 可减 轻与电解质的界面反应, 达到提高库伦效率、提高循环 寿命、提高锂电池安全性的作用.

使用 $\mathrm{HCE}$ 的 $\mathrm{NMC} / \mathrm{Li}$ 电池虽然具有较高的库伦效 率, 电压稳定, 但放电容量较低, 仅为 $140 \mathrm{mAh} / \mathrm{g}$, 电极 极化明显增加, 且持续衰减, 100 次循环后仅保留 76\% 的容量, 原因归结为高粘度、低导电性和低润湿性等导 致的电极在高电流密度下反应动力学迟缓. 与之形成鲜 明对比的是, 使用 $\mathrm{LHCE}$ 的 $\mathrm{NMC} / \mathrm{Li}$ 电池放电容量为 $150 \mathrm{mAh} / \mathrm{g}$, 具有良好的长循环稳定性(300 次循环后容 量保持率为 $95 \%$ ), 平均库伦效率为 $99.7 \%, 0.5 \mathrm{C}$ 充电 2 $\mathrm{C}$ 放电时, 仍可提供较高放电比容量 $(150 \mathrm{mAh} / \mathrm{g})$, 即使 在 700 次循环后仍可保持 $80 \%$ 的容量.

通过前线轨道理论计算结合拉曼光谱(Raman)、核 磁共振波谱(NMR) 分析上述局部高浓度电解质发现: LiFSI 与稀释剂 BTFE 之间的作用力远弱于 LiFSI 与溶 剂 DMC 之间的作用力(图 4); 同时 BTFE 相比其他组分, 具有对锂金属更高的稳定性. 另外, XPS 证实: $\mathrm{FSI}^{-}$会优 先在锂负极表面发生分解, 产生 $\mathrm{LiF}$ 含量更高、更稳定 的 SEI 膜, 从而进一步稳定金属锂/电解液的界面, 提升 库伦效率和循环稳定性. 此外, 稀释剂 BTFE 的加入能 够提高 $\mathrm{Li}^{+}$的扩散能力, 提高离子电导率, 改善电池的 倍率和低温性能. 因此, 采用 $\mathrm{LHCE}$ 的 $\mathrm{LiNi}_{1 / 3} \mathrm{Mn}_{1 / 3}-$ $\mathrm{Co}_{1 / 3} \mathrm{O}_{2} / \mathrm{Li}$ 电池循环 700 圈后, 容量保持率高于 $80 \%$, 库 伦效率达 $99.5 \%$.

砜类化合物作为溶剂, 表现出独特的抗氧化稳定 性. 此外, 砜还具有高介电常数和低可燃性, 这两者都 是锂电池应用所需要的. 但由于其与锂金属负极相容性 差、粘度大、润湿性差等缺点, 限制了其在锂金属电池 中的应用. 2018 年, 张继光和许武等 ${ }^{[45]}$ 通过加入能与砜 类溶剂混溶但是不与锂盐结合的氟代醚类 TTE, 对环丁 砜(TMS)高浓度电解液进行稀释, 得到的局域高浓度电

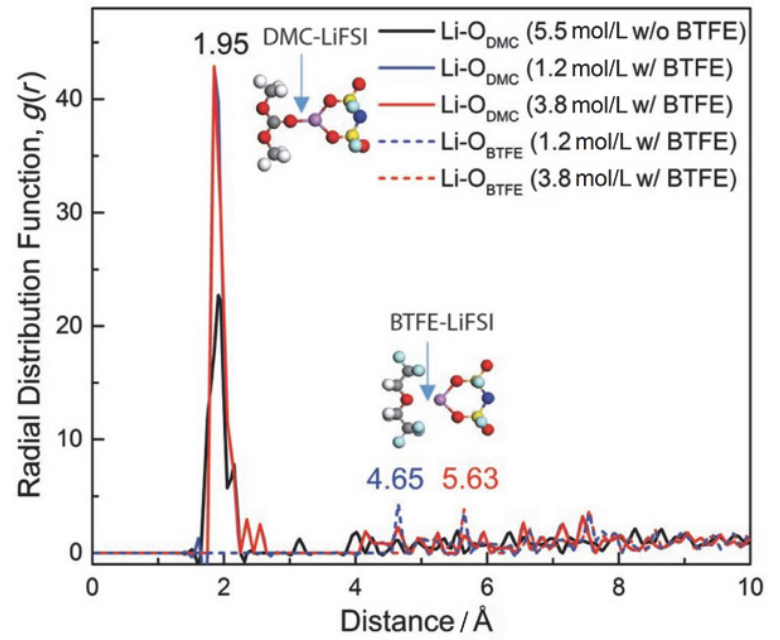

图 $430{ }^{\circ} \mathrm{C}$ 下 $\mathrm{Li}-\mathrm{O}_{\mathrm{DMC}}$ 和 $\mathrm{Li}-\mathrm{O}_{\mathrm{BTFE}}$ 的径向分布函数图, DMC-LiFSI 和 BTFE-LiFSI 溶剂-盐结构图(插入图)

Figure 4 Radial distribution functions of $\mathrm{Li}-\mathrm{O}_{\text {DMC }}$ and $\mathrm{Li}-\mathrm{O}_{\text {BTFE }}$ pairs calculated from AIMD simulation trajectories at $30{ }^{\circ} \mathrm{C}$, with insets showing the structures of DMC-LiFSI and BTFE-LiFSI solvent-salt pairs

解质(LiFSI-3TMS-3TTE), 首次实现了砜类电解液在低 温下 $\left(-10{ }^{\circ} \mathrm{C}\right)$ 的使用. 在不同温度下对 $\mathrm{LiNi}_{0.6} \mathrm{Mn}_{0.2^{-}}$ $\mathrm{Co}_{0.2} \mathrm{O}_{2} / \mathrm{Li}$ 电池进行测试, 发现局部高浓度电解质能表 现出更好的倍率性能.

稀释剂 TTE 的加入能够显著地解决砜基电解质润 湿性差的问题，使电解质很容易地分散和浸润聚乙烯隔 膜、正极和负极表面. 使用局部高浓度电解质并在不同 温度下对 $\mathrm{LiNi}_{0.6} \mathrm{Mn}_{0.2} \mathrm{Co}_{0.2} \mathrm{O}_{2} / \mathrm{Li}$ 电池进行测试, 电池在 不同温度下均表现出较好的倍率性能. 当无稀释剂时, 电池不能在 $0{ }^{\circ} \mathrm{C}$ 下运行, 但局部高浓度电解质 (LiFSI-3TMS-3TTE)仍然可以在 $1 \mathrm{C}\left(1.5 \mathrm{~mA} / \mathrm{cm}^{2}\right)$ 或 $2 \mathrm{C}$ 倍率 $\left(0{ }^{\circ} \mathrm{C}\right.$ 甚至 $\left.-10{ }^{\circ} \mathrm{C}\right)$ 下保留大部分电池容量, 表明, 将砜类电解液局部高浓化后可以显著提高其实际应用 的可行性. SEM 观察到, 使用局部高浓度电解质后, 锂 金属沉积得到明显改善(图 5), 且首圈的库伦效率由 83.0\%提高到 $97.3 \%$, 并且这种基于砜类的 LHCE 能显 著抑制锂负极与电解质的反应，促进高性能 SEI 层的生 成, 实现高效的锂金属循环(库伦效率平均达到 98.8\%).

除以上性能外, LHCE 还能够解决高浓度砜类电解 液在高压下的铝箔集流体腐蚀问题，最终使 $\mathrm{LiNi}_{0.6} \mathrm{Mn}_{0.2} \mathrm{Co}_{0.2} \mathrm{O}_{2} / \mathrm{Li}$ 金属电池在 $4.9 \mathrm{~V}$ 高电位下得以稳 定循环. 此研究不仅为砜基电解质应用于锂金属负极或 其他高容量负极材料提供了创新性的研究方法, 而且为 提高电池在高压条件下的循环性能奠定了基础.

2018 年，何向明等 ${ }^{[46]}$ 通过加入 “辅助溶剂”进行“模 拟高浓度电解质” 的探索, 通过向高浓度 LiTFSI 电解液 中加入 HFME 获得局部高浓度电解质 $[n$ (LiTFSI)/ $n(\mathrm{DMC})=1: 1.5]$, HFME 约占电解质的 $50 \%$. 从外观上 看，局部高浓度电解质是稳定、透明、均匀的溶液. 线 性扫描伏安(LSV)数据表明高浓度电解质和局部高浓度 


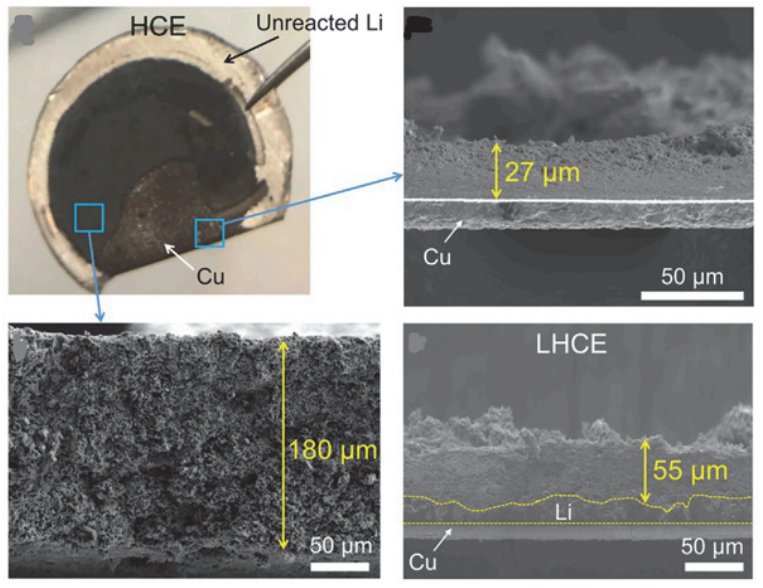

图 5 锂负极在 HCE 中循环 300 圈后的 SEM 图和在 LHCE 中循环 300 圈后的 SEM 截面图

Figure 5 The SEM image of Li anode cycled in the HCE for 300 cycles and the cross-section SEM image of the Li anode cycled in the LHCE for 300 cycles

电解质的氧化电位均为 $5.65 \mathrm{~V}$, 这表明加入稀释剂保持 了原高浓度电解质宽电化学窗口的特点. 并且高浓度电 解质和局部高浓度电解质都可以有效地抑制铝箔腐蚀. 分别使用高浓度电解质和局部高浓度电解质组装电池, 测试数据表明: 二者对比, 使用局部高浓度电解质后, 电池具有较高的离子电导率(高浓度电解质的 3.4 倍)和 较低的粘度(高浓度电解质的 0.028 倍). 使用局部高浓 度电解质组装的 $\mathrm{LiNi}_{1 / 3} \mathrm{Co}_{1 / 3} \mathrm{Mn}_{1 / 3} \mathrm{O}_{4}$ /石墨电池, 循环 500 次后, 容量保持率为 $89.2 \%$, 高于稀电解质 $(79.2 \%)$ 和高 浓度电解质(86.2\%). 此局部高浓度电解质具有优异循 环性能的原因为: 局部高浓度电解质离子电导率高, 电 解质润湿性好, 抗氧化能力强.

为解决醚类电解质不耐高电压这一问题, 2019 年, 张继光等 ${ }^{\left[{ }^{[7]}\right.}$ 设计了一种基于醚类溶剂的局部高浓度电 解质, 显著提高了其电化学稳定性. 其制备过程是: 将 LiFSI 与 DME 按物质的量比 $1: 1.2$ 混合后, 加入 TTE, 得到局部高浓度电解质. 这样既保持了高浓度电解质原 有的溶剂化结构 $[n(\mathrm{LiFSI}): n(\mathrm{DME}): n(\mathrm{TTE})=1$ : $1.2: 3$ ] 和性质, 又降低了电解液粘度, 成功解决了梄类 在高电压下稳定性差的难题. 氟醚稀释剂的加入不仅在 粘度、润湿性、成本等方面具有良好的改善作用, 而且 使正极和负极上形成有效的 SEI 抑制不良副反应. 在高 电压、高负载量正极、薄锂负极的严格条件下, 采用该 LHCE 电解液表现出高能量密度 $325 \mathrm{Wh} / \mathrm{kg}$ (根据扣式电 池的电极材料、集流体、隔膜和电解质, 不包括扣式电 池外壳的质量). 文章认为, 将此局部高浓度电解质制 成真正的软包电池时, 使用铝塑膜包装, 电池重量将在 一定程度上减少, 且采用多层电极卷绕或叠片等手段. 因此, 软包电池的能量密度可能会高于扣式电池. 使用 高面密度 $\left(4.2 \mathrm{mAh} / \mathrm{cm}^{2}\right)$ 的三元正极, 添加 $14 \mu \mathrm{L}$ 电解液, 组装电池循环 155 圈后, 依然能够保持 $80 \%$ 的容量. 并 且此局部高浓度电解质可匹配 $4.5 \mathrm{~V}$ 级三元正极
(NMC811), 当使用铜箔代替锂负极时，组装无锂负极 电池，电池循环 70 圈后依然保持 $77 \%$ 的初始比容量. 这 一醚类 LHCE 在实用条件下对锂负极和高载量正极的 稳定性是非常突出的. 这一突破性成果为未来高电压锂 金属电池电解液的研发提供了重要的思路, 标志着高电 压锂金属电池向着实用化又迈进了一步.

\section{4 局部高浓度电解质在低温二次锂电池中的应 用}

商用锂离子电池电解液一般使用链状碳酸酯类溶 剂, 熔点较高(如碳酸乙烯酯, $35 \sim 38{ }^{\circ} \mathrm{C}$; 碳酸二甲酯, $\left.2 \sim 4{ }^{\circ} \mathrm{C}\right)$, 限制了其在低温, 特别是 $-40{ }^{\circ} \mathrm{C}$ 以下的实 际应用. 为了实现可充电电池的低温运行, 人们对电解 液配方进行了大量的改进, 如混合溶剂、新型盐和电解 液添加剂、新型溶剂等. 最近, 乙酸乙酯(EA)被报道在 $-70{ }^{\circ} \mathrm{C}$ 的超低温度下实现促进可充电锂电池的操作, 因为它比传统溶剂的熔点低得多. 但是, 它电化学窗口 较窄, 严重阻碍了其在高电压锂电池中的应用. 高浓度 电解质能够有效提高氧化稳定性, 但是高浓度必然导致 低温下黏度的大幅度增加. 因此如何设计一种满足对锂 金属稳定且可以匹配高电压正极的低粘度和高离子电 导率的电解液, 是实现低温高电压二次电池运行的关键 一步.

为解决这一问题, 2019 年, 夏永姚等 ${ }^{[48]}$ 通过向高浓 度电解液中引入低粘度的电化学惰性的稀释剂, 得到局 部高浓电解液体系 $(5 \mathrm{~mol} / \mathrm{L} \mathrm{LiTFSI} / \mathrm{EA}+$ 二氯甲烷 (DCM), 体积比 $1: 4)$. 该电解质保留了宽电化学窗口 的优点, 并解决了高浓度电解质高粘度的缺点. 在溶剂 足够的低浓度电解液中, 多余的溶剂分子可以取代 $\mathrm{TFSI}^{-}$的束缚形成 SSIPs (solvent-separated ion pairs)结 构. 将溶剂的体积逐渐减少，电解质的浓度升高形成高 浓度电解质，电解质体系中溶剂化络合物也以不同的形 式共存, 包括两种：接触离子对 CIPs (contaction pairs, 一个 $\mathrm{FSI}^{-}$配一个 $\mathrm{Li}^{+}$); 聚集体 AGGs (cation-anion aggregates 一个 $\mathrm{FSI}^{-}$配两个或更多 $\mathrm{Li}^{+}$, 没有自由的 $\mathrm{FSI}^{-}$). 合适的稀释剂加入, 可以直接降低高浓度电解质的浓 度, 但挑战在于, 稀释剂应对高浓度电解质的溶剂化结 构影响较小, 甚至没有影响. 与高浓度电解质的溶剂化 结构相同, 在分子水平上看, 局部高浓度电解质可以看 作是高浓度电解质与稀释剂的混合物. 文章通过能谱表 征和原子动力学模拟, 证明了该体系加入稀释剂后仍然 与高浓度电解质的溶剂化结构相同(图 6).

使用该体系可以在 $-70{ }^{\circ} \mathrm{C}$ 的超低温度下运行, 具 有高电位窗口 $(4.85 \mathrm{~V})$, 高离子电导率 $(0.6 \mathrm{mS} / \mathrm{cm})$, 低 粘度 $(0.35 \mathrm{~Pa} \bullet \mathrm{s})$ 等特性. 基于这种局部高浓度电解质, 采用 PI 有机聚酰亚胺正极的电池可在 $-70{ }^{\circ} \mathrm{C}$ 下提供高 能量密度 $\left(178 \mathrm{Wh} \bullet \mathrm{kg}^{-1}\right)$ 和功率密度 $\left(2877 \mathrm{~W} \cdot \mathrm{kg}^{-1}\right)$. 这种 局部高浓度电解质的设计不仅保证了优异的电化学性 


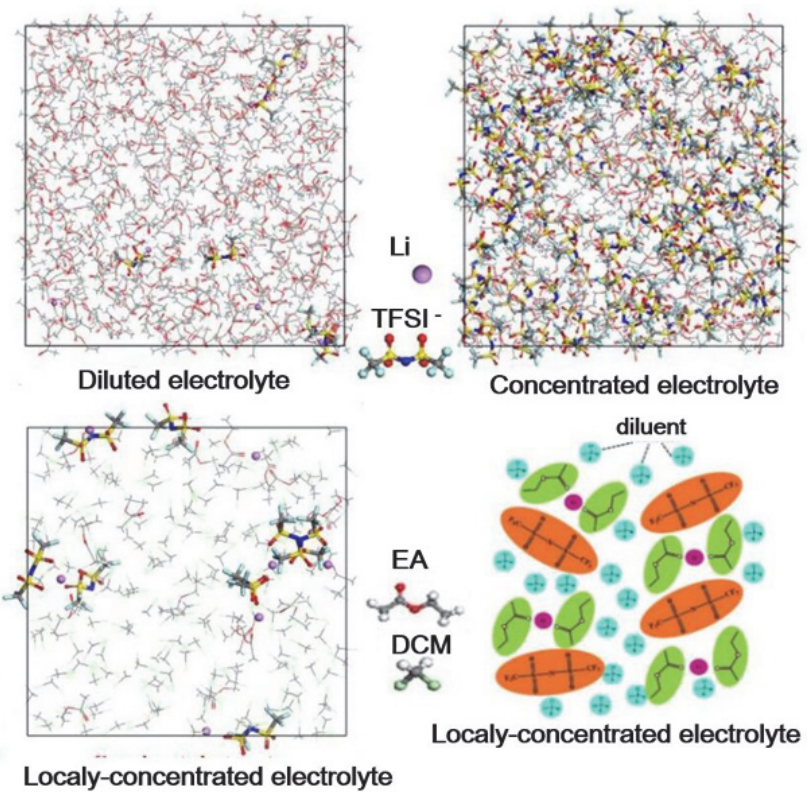

图 6 低浓度电解质 $(0.15 \mathrm{~mol} / \mathrm{L})$ 、高浓度电解质 $(5 \mathrm{~mol} / \mathrm{L})$ 和局部高浓 度电解质 $(5 \mathrm{~mol} / \mathrm{L}-1-4) \mathrm{MD}$ 计算图

Figure 6 Snapshots of MD (molecular dynamics) simulation boxes for diluted $(0.15 \mathrm{~mol} / \mathrm{L})$, concentrated $(5 \mathrm{~mol} / \mathrm{L})$ and locally-concentrated $(5$ $\mathrm{mol} / \mathrm{L}-1-4)$ electrolyte

能, 还为极端工作温度下的高能量密度电池体系提供了 新的思路. 但是, 二氯甲烷具有较低的沸点 $\left(39.8{ }^{\circ} \mathrm{C}\right)$, 几乎在室温下就可以挥发, 难以用于高温二次电池当 中. 所以科研工作者还需要继续探究新型的电解液体 系, 需要进一步优化电解质溶剂和添加剂, 在宽温区 (高低温)范围内都可以实现平稳运行.

\section{5 局部高浓度电解质在抑制锂硫电池多硫化物 穿梭中的应用}

不同于锂离子电池, 锂硫电池是以硫单质元素作为 正极, 金属锂作为负极的一种二次电池 ${ }^{[49-51]}$. 利用硫作 为正极材料的锂硫电池的优势在于: 硫的理论比容量和 电池理论质量能量密度分别达到 $1675 \mathrm{mAh} / \mathrm{g}$ 和 2600 $\mathrm{Wh} / \mathrm{kg}$, 远高于目前商业化的锂离子电池. 同时单质硫 在地球中储量丰富, 并且石油冶炼的副产物就能提供丰 富的单质硫, 因此单质硫的价格非常低. 硫极易升华, 简单的加热就可以将正极中硫进行回收, 未来废旧锂硫 电池的回收再利用相对简单, 是一种环境友好、极具应 用前景的电化学储能体系 ${ }^{[52 \sim 54]}$.

然而, 锂硫电池在充放电过程中产生的可溶性多硫 化合物, 会导致 “穿梭效应”: 充放电过程中, 正极产生 的多硫化物 $\left(\mathrm{Li}_{2} \mathrm{~S}_{x}\right)$ ) 容解到电解液中, 并穿过隔膜, 向负 极扩散，与负极的金属锂直接发生反应，最终造成了电 池中有效物质的不可逆损失、电池寿命的衰减、库伦效 率较低等问题, 甚至会引发一系列安全问题 ${ }^{[55 ~ 57]}$.

2014 年, Nazar 课题组 ${ }^{[58]}$ 报道了将局部高浓度电解 质用于锂硫电池抑制多硫化物穿梭效应的文章. 在局部
高浓度电解质中, 所有的乙腈(ACN)均与 LiTFSI 结合在 一起, 形成 $(\mathrm{ACN})_{2} \cdot \mathrm{LiTFSI}$ 络合物, 这不仅阻止了溶剂 与金属锂的反应，同时还抑制了溶剂对多硫化物的溶解 (图 7).

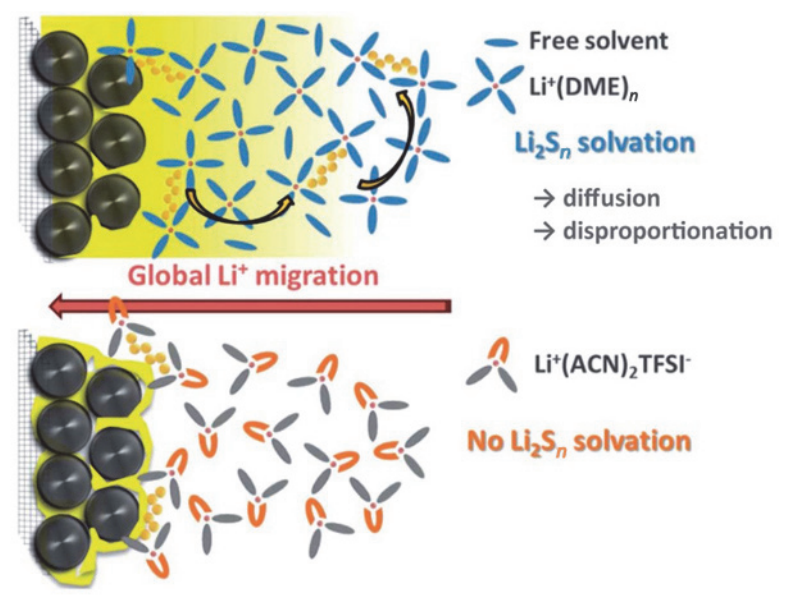

图 7 锂硫电池中多硫化物的去溶剂化概念图

Figure 7 Concept of a nonsolvent for polysulphides in a Li-S battery

为了降低粘度, 文中将 $(\mathrm{ACN})_{2} \mathrm{LiTFSI}$ 与 HFE 共混 形成局部高浓度电解质[(ACN $)_{2}$-LiTFSI]-HFE. HFE 的氟 化程度太高, 无法参与 $\mathrm{Li}^{+}$的溶剂化, 同时 HFE 也无法 溶解多硫化物, 因此加入 HFE 还可以将多硫化物的溶 解度降到最低. 文章通过阻抗、极化和电池容量保持率 等方面进行了 [(ACN $)_{2}$-LiTFSI]-HFE 体积比的优化; 还 通过 X 射线吸收光谱(XANES)等电化学方法证明了多 硫化锂是在 $(\mathrm{ACN})_{2}$-LiTFSI 中形成, 但其溶解度几乎是 可以忽略不计的, 但它们表现出极其有限的流动性, 这 对电池性能产生了显著影响: 在小倍率 $0.2 \mathrm{C}$ 的条件下, 电池首圈放电比容量可以达到 $1400 \mathrm{mAh} \cdot \mathrm{g}^{-1}$.

同样使用对锂盐和多硫化物不溶解的 HFE 作为共 溶剂, 成功解决了锂硫电池的多硫化物穿梭的瓶颈问 题, 2015 年 Dokko 课题组 ${ }^{[59]}$ 采用脉冲场梯度自旋回波 (PGSE)核磁共振和 Raman 分析了 G3、HFE 和 $1 \mathrm{~mol} / \mathrm{L}$ 的双三氟甲烷磺酰亚胺锂 Li[TFSA]组成的局部高浓度 电解质中 $\mathrm{Li}^{+}$的溶剂化结构和离子对的形成. 研究发现: 由于 HFE 介电常数相对较低, 所以它几乎不参与溶剂 化过程, $\mathrm{Li}^{+}$在电解液中被 $\mathrm{G} 3$ 溶解, 形成一个 $[\mathrm{Li}(\mathrm{G} 3)]^{+}$ 络合物阳离子. 随着 G3/Li[TFSA]物质的量比的降低, $\mathrm{Li}[\mathrm{TFSA}]$ 在溶液中的解离性降低. 当 $\mathrm{G} 3$ 与 $\mathrm{Li}^{+}$物质的量 比接近 $1: 1$ 时, 电解质中 $\mathrm{G} 3$ 几乎全部与锂盐配位, 电 解质的氧化稳定性增强, 加入稀释剂 HFE 形成 $1 \mathrm{~mol} / \mathrm{L}$ 的局部高浓度电解质，能起到抑制正极铝集流体腐蚀的 作用, 使 $4 \mathrm{~V}$ 的锂电池能够稳定运行. G3 含量的多少也 影响了锂硫电池电化学反应过程, 锂硫电池反应中间产 物为多硫化锂, 在 $n(\mathrm{G} 3) / n(\mathrm{Li}[\mathrm{TFSA}])=1$ 的局部高浓度 电解质中, $\mathrm{Li}_{2} \mathrm{~S}_{m}$ 的溶解微乎其微, 因而能实现锂硫电池 高效稳定充放电. 
离子液体也被报道能起到抑制多硫化物穿梭的作 用, 1980 年, 离子液体首次作为电池的电解质使用. 此 后的几十年, 人们对于离子液体作为电解质前景愈加看 好, 离子液体作为锂电池体系电解液的研究也越来越 多. 与一般的有机电解液相比, 离子液体电解液具有宽 温度范围、低蒸气压、不燃性、无着火点、高热稳定性、 宽电化学窗口等诸多优点, 用作电解质可以显著提高锂 电池的安全性 ${ }^{[60 ~ 64]}$. 但是离子液体作为电解液黏度太 高, 其黏度主要由氢键和范德华力二者共同来决定, 比 一般的有机溶剂高 $1 \sim 2$ 个数量级. 为解决这一难题, 2013 年 Watanabe 课题组 ${ }^{[65]}$ 用 HFE 为稀释剂得到的局部 高浓度离子液体电解质, 并用于锂硫电池抑制其穿梭效 应, 从而提高锂硫电池的循环稳定性。在 $\left[\mathrm{Li}(\text { glyme })_{x}\right][\mathrm{TFSA}]$ 中, $\mathrm{Li}_{2} \mathrm{~S}_{m}$ 的溶解度随着乙二醇二甲 醚 $\left((\text { glyme })_{x}\right)$ 物质的量比的降低而降低. 其原因主要归 结为: 在高浓度离子液体电解质中, 所有的溶剂分子都 与 $\mathrm{Li}^{+}$配位, 因此没有游离的溶剂分子. 同时氧原子上 所有的孤对电子都给予了 $\mathrm{Li}^{+}$, 导致对亲电子的 $\mathrm{Li}_{2} \mathrm{~S}_{m}$ 溶 解度很差, 从而抑制了多硫化物溶解, 库伦效率得到明 显提升.

以上述工作为基础, 2015 年, 该课题组 ${ }^{[66]}$ 采用扩散 率测量和拉曼光谱法研究了锂盐在使用不同稀释剂的 局部高浓度离子液体电解质中的溶解度. 结果表明: 文 中使用的稀释剂, 如水、丙烯腈 $(\mathrm{AN})$ 、丙酮、DEC 与锂 金属会发生反应，而 PC 会与络合物阳离子发生不利的 配体交换反应. 但甲苯或 HFE 稀释后的离子液体电解 质 $[\mathrm{Li}(\mathrm{G} 4)][\mathrm{TFSA}]$ 离子电导率较高, 可作为二次电池电 解质使用.

总体来讲: 以上工作首先制备出一种能适用于锂硫 电池的高浓度电解质, 然后加入高氟化、低极性、不溶 解锂盐且不溶解多硫化物的氟醚来制备并得到局部高 浓度电解质. 当溶液中的锂盐与溶剂达到饱和后, 络合 形成[溶剂-LiTFSI]-HFE 的溶剂化结构. 局部高浓度电 解质接近饱和的 [溶剂-LiTFSI]-HFE 不能提供多余的溶 剂分子来溶解多硫化物, 从而阻止了多硫化物其与负极 反应. 因此上述工作既能起到抑制锂硫电池中多硫化物 溶解穿梭, 也能提升界面反应动力学, 稳定理负极、抑 制锂枝晶的生成, 从而大幅度提高电池充放电库仑效 率. 但是文章中使用的稀释剂均为 HFE, 种类相对比较 单一, 我们思考: (1)是否可以探索和采用更多其他类别 的稀释剂种类, 使电池拥有更优异的循环性能? (2) 是 否可以添加聚合单体制成局部高浓度凝胶型聚合物电 解质, 在兼顾抑制多硫化物穿梭的功能上, 进一步提升 锂硫电池的安全性能?

\section{6 局部高浓度电解质在高性能二次钠电池中的 应用}

钠离子电池与锂离子电池具有相似的架构和工作
原理. 考虑到钠较高的理论比容量 $\left(1165 \mathrm{mAh} \cdot \mathrm{g}^{-1}\right)$ 和较 低的氧化还原电位( $2.714 \mathrm{~V}$ vs 标准氢电极), 同时钠资 源丰富、价格低廉, 钠离子电池是未来锂离子电池的十 分理想的替代品, 在规模化电能存储系统中具备强大的 竞争优势. 然而, 钠金属与液态电解质之间反应活泼, 导致充放电库仑效率较低(一般为 $95 \%$ ), 进一步引起钠 金属电池容量快速衰减，这在很大程度上阻碍了金属钠 作为二次电池负极的实际应用 ${ }^{[6,67 \sim 71]}$.

近年来, 高浓度电解质由于其独特的溶剂化结构和 功能在二次锂金属电池中得到了较为广泛的应用, 这也 同样适用于二次钠金属电池. 张继光等 ${ }^{[21]}$ 以 $4 \mathrm{~mol} / \mathrm{L}$ 双 (氟磺酰)亚胺钠(NaFSI)和醚类溶剂(1,2-二甲氧基乙烷、 $\mathrm{DME}$ )为基础制备出高浓度电解质, 实现了 $\mathrm{Na}$ 的可逆沉 积与溶出. 然而, 高浓度电解质粘度高, 对隔膜润湿性 差, 而且盐的成本较高, 距离其大规模应用还有很大的 挑战.

2019 年, 张继光团队 ${ }^{[33]}$ 首次将局部高浓度电解质 用于钠离子电池, 为克服 HCEs 的缺点, 他们采用介电 常数低、反应位点少的 “惰性” 氟代醚溶剂将 HCE 稀 释, 形成 LHCE. 局部高浓电解液选用 BTFE 为稀释剂, 制成具有较低的总盐浓度 $(2.1 \mathrm{~mol} / \mathrm{L} \mathrm{NaFSI} / \mathrm{DME}-\mathrm{BTFE})$ $[n(\mathrm{DME}): n(\mathrm{BTFE})=1: 2]$ 局部高浓度电解质.

“惰性” 稀释剂 BTFE 对高浓度电解质中的阳离 子-阴离子团聚体(AGGs)的溶剂化结构几乎没有影响. 而且，它可以显著降低钠盐浓度，降低电解质体系粘度， 增加离子电导率，同时提高电解质对正负极的润湿性. 实验结果证明: 组装的 $\mathrm{Na}_{3} \mathrm{~V}_{2}\left(\mathrm{PO}_{4}\right)_{3} / \mathrm{Na}$ 金属电池, 能够 在 $20 \mathrm{C}$ 的倍率下循环 40000 次后容量保持率达到 $90.8 \%$, 库伦效率 $>99 \%$, 并且无钠枝晶产生, 极大地提 高了钠金属电池的性能(图 8). 稀释剂 BTFE 的加入, 既 不会破坏 $\mathrm{Na}^{+}-\mathrm{FSI}^{-}-\mathrm{DME}$ 的溶剂化结构, 又对改善 $\mathrm{Na}$ 金属负极的界面反应动力学和界面稳定性起着至关重 要的作用. 这种局部高浓度电解质的性能明显优于高浓 度电解质 (5.2 mol/L NaFSI/DME). 此项工作为开发高性 能钠离子电池新型电解质开辟了新的途径; 同时也为其 他新型二次金属电池(如钾金属电池)用新型电解质的开 发, 提供了关键理论指导和重大借鉴.

\section{7 总结与展望}

局部高浓度电解质, 既保留了传统高浓度电解质的 优势, 如宽电化学窗口、高倍率性能、高安全性、高离 子迁移数、抑制铝集流体腐蚀、抑制锂枝晶生长、形成 优异的固体电解质界面等优势，同时又解决了高浓度电 解质体系实际应用所面临的诸多难题(如高粘度、高成 本、对电极和隔膜浸润性差等问题). 鉴于局部高浓度电 解质的这些优点，截止到目前，局部高浓度电解质已经 在阻燃二次电池、高电压二次锂电池、低温二次锂电池、 锂硫电池和钠电池等方面取得了阶段性科研进展, 且效 


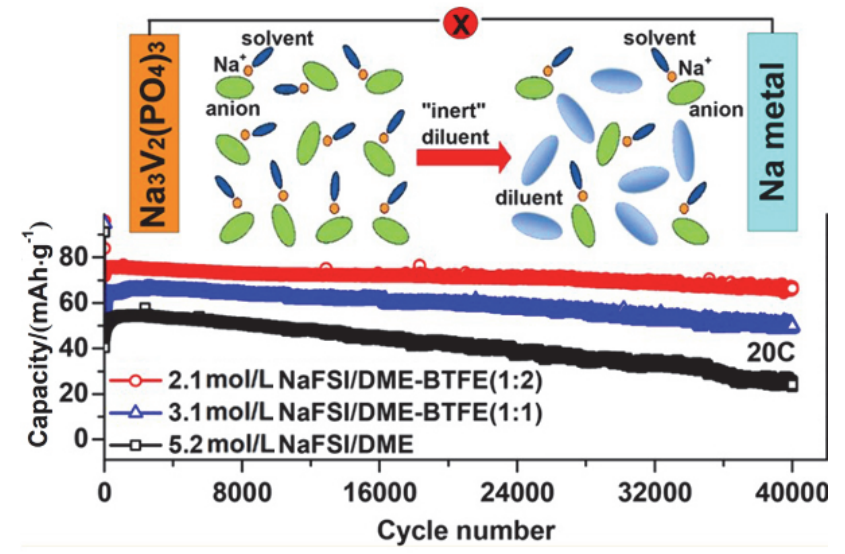

图 8 局部高浓度电解质在高性能二次钠电池中的工作性能及原理图 Figure 8 The working principle and performance of localized high concentration electrolyte in high performance sodium ions battery

果显著, 并极有可能在未来高性能二次电池领域充当重 要角色.

尽管如此，局部高浓度电解质的应用仍然面临诸多 挑战: (一) 局部高浓度电解质, 可以显著降低成本和电 解质粘度, 但其对正负极的浸润性, 有待进一步的验 证. 通过同时解决成本、粘度、浸润性等相关技术壁垒, 可以匹配更多候选电极材料, 可以为先进电池系统突破 打下坚实基础. (二)目前, 局部高浓度电解质中稀释剂 种类仅仅局限于以 HFE 和 BTFE 为代表的氟代醚类(表 1), 并未能应用于高温二次电池当中, 种类较为单一, 沸点低且比较容易挥发, 将局部高浓度电解质的使用范 围限制在低温区, 难以满足更高安全性能电解质的苛刻 要求, 因此实现全温区的局部高浓度电解质二次电池还 需要进一步的探究, 还需要不断地探索新的稀释剂, 采 用更多的低极性溶剂, 如: 甲苯和烷类等, 并将现有的 氟代醚类稀释剂种类进行增添拓展，如 T5202，T3057, FE2, T1216(图 9) ${ }^{[72]}$. 新型稀释剂的选择应当满足以下 特征: (1)为 “情性” 溶剂, 低极性. 在不改变高浓度电解 液独特的溶剂化结构的同时, 还能够与高浓度电解质互 溶; (2)廉价, 成本低, 能够商业化推广应用; (3)低粘度;

(4)电化学稳定性好; (5)阻燃, 低挥发性; (6)不引发降低 电池性能的副反应; (7)与正负极具有良好的相容性, 能 够形成优异的固态电解质膜. (三)目前对于局部高浓电 解质中稀释剂的作用机制以及所形成的络合分子结构, 通常是使用拉曼光谱和理论计算(如分子动力学模拟方 法)来进行探究, 方法较为单一, 未能有其它测试手段 方法. 可以针对局部高浓度电解质内部以及原子尺度、 原子数量、络合结构进行更加清楚深入的探究, 这将更 加有助于让我们了解到潜在的原子尺度相互作用下优 异的固态电解质层、高离子电导率、高离子迁移数、宽 电化学窗口等特殊性能的作用机制. (四)局部高浓度电 解质能够有效抑制铝集流体腐蚀、形成独特高性能的 SEI、抑制锂枝晶等作用，通常采用 SEM 和 XPS 进行表 征, 缺乏原位测试手段的表征测试, 发展原位测试手段 将更有助于我们了解局部高浓度电解质在电池循环过 程内部的作用机制.

总体来讲, 局部高浓度电解质的出现为开发和研究 高性能二次电池开辟了一条新途径; 同时也为钠金属电 池、钾金属电池、镁金属电池等其它新型二次电池系统 新型电解质的开发, 提供了非常好的理论指导和思路. 相信在不久的未来, 局部高浓度电解质必将在高性能二 次电池领域得到蓬勃发展和产业应用.

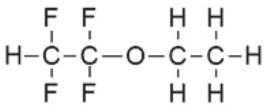

$$
\begin{aligned}
& \text { 1,1,2,2-tetrafluoroethyl } \\
& \text { ether } \\
& \text { (T5202) } \\
& \mathrm{F} \mathrm{F} \mathrm{H} \mathrm{F} \mathrm{H} \mathrm{F} \\
& \mathrm{H}-\mathrm{C}-\mathrm{C}-\mathrm{C}-\mathrm{O}-\mathrm{C}-\mathrm{C}-\mathrm{C}-\mathrm{F}
\end{aligned}
$$

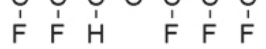

1,1,2,3,3,3-hexafluoropropyl2,2,3,3-tetrafluoropropyl ether (FE2)

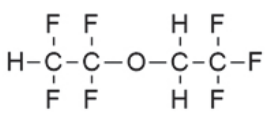

1,1,2,2-tetrafluoroethyl-2,2,2trifluoroethyl ether (T3057)

$$
\begin{aligned}
& \mathrm{FF} \quad \mathrm{HFF} \\
& \mathrm{H}-\mathrm{C}-\mathrm{C}-\mathrm{O}-\mathrm{C}-\mathrm{C}-\mathrm{C}_{1}-\mathrm{C}-\mathrm{F}
\end{aligned}
$$

2,2,3,3,3-pentafluoropropyl-

\begin{tabular}{|c|c|c|c|c|c|}
\hline 电解质(盐、主要溶剂、添加剂等) & 稀释剂 & 电极材料 & 循环圈数 & 保持率 & Ref. \\
\hline $1.2 \mathrm{~mol} / \mathrm{L} \mathrm{LiFSI} / \mathrm{TEP}+\mathrm{BTFE}(1: 3$ by mol $)$ & BTFE & $\mathrm{Li} \| \mathrm{NMC}_{622}$ & 600th & $>97 \%$ & {$[32]$} \\
\hline $1 \mathrm{~mol} / \mathrm{L} \mathrm{LiFSI} / \mathrm{OFE}+\mathrm{DME}(95: 5$ by volume $)$ & OFE & $\mathrm{Li} \| \mathrm{C} / \mathrm{S}$ & 150th & $>65 \%$ & {$[41]$} \\
\hline $1.2 \mathrm{~mol} / \mathrm{L} \mathrm{LiFSI} / \mathrm{TEP}+\mathrm{FEC}+\mathrm{BTFE}(1.2: 0.13: 4 \mathrm{by} \mathrm{mol})$ & BTFE & $\mathrm{Si} \| \mathrm{NMC}_{111}$ & 600th & $>90 \%$ & {$[42]$} \\
\hline $1.2 \mathrm{~mol} / \mathrm{L} \mathrm{LiFSI} / \mathrm{DMC}+\mathrm{BTFE}(1: 2$ by mol $)$ & BTFE & $\mathrm{Li} \| \mathrm{NMC}_{111}$ & 700th & $>80 \%$ & [44] \\
\hline LiFSI/3TMS-3TTE (by mol) & TTE & $\mathrm{Li}|| \mathrm{NMC}_{111}$ & 300th & $>81 \%$ & [45] \\
\hline $2.5 \mathrm{~mol} / \mathrm{L}$ LiTFSI/DMC + HFME ( $1: 1$ by volume $)$ & HFME & graphite $\| \mathrm{NMC}_{111}$ & 500 th & $>90 \%$ & [46] \\
\hline LiFSI/1.2DME-3TTE (by mol) & TTE & $\mathrm{Li} \| \mathrm{NMC}_{811}$ & 150 th & $>80 \%$ & [47] \\
\hline $5 \mathrm{~mol} / \mathrm{L}$ LiTFSI/EA+DCM ( $1: 4$ by volume $)$ & DCM & $\mathrm{Li} \| \mathrm{PI}$ & 100th & $>69 \%$ & {$[48]$} \\
\hline $1 \mathrm{~mol} / \mathrm{L} \mathrm{LiTFSI} / \mathrm{G} 3+4.46 \mathrm{HFE}$ (by mol) & HFE & $\mathrm{LiC} \| \mathrm{S}-\mathrm{KB}$ & 100th & $>77 \%$ & [59] \\
\hline$\left[(\mathrm{ACN})_{2} / \mathrm{LiTFSI}\right]+\mathrm{HFE}(1: 1$ by volume $)$ & HFE & $\mathrm{Li} \| \mathrm{C} / \mathrm{S}$ & 100th & $>74 \%$ & {$[58]$} \\
\hline LiTFSA/G4+HFE $(1: 1: 4$ by mol $)$ & HFE & $\mathrm{Li} \| \mathrm{C} / \mathrm{S}$ & 50 th & $>90 \%$ & {$[65]$} \\
\hline $2.1 \mathrm{~mol} / \mathrm{L} \mathrm{NaFSI} / \mathrm{DME}+\mathrm{BTFE}(1: 2$ by mol $)$ & BTFE & $\mathrm{Na}|| \mathrm{Na}_{3} \mathrm{~V}_{2}\left(\mathrm{PO}_{4}\right)_{3}$ & 4000th & $>90 \%$ & {$[33]$} \\
\hline
\end{tabular}
1,1,2,2-tetrafluoroethyl ether (T1216)

图 9 氟代醚类稀释剂结构图

Figure 9 Molecular structures of fluoroalkyl ether as a diluent

表 1 局部高浓度电解质的组成和循环性能对照表

Table 1 Comparison of composition and cycling performance for localized high-concentration electrolytes 


\section{作者简介}

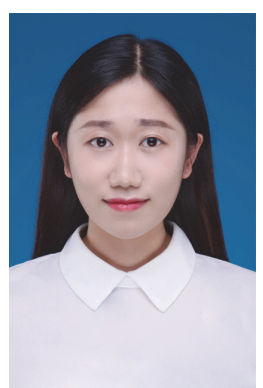

于喆, 女, 青岛科技大学化学与分子工程学院硕士, 中国 科学院青岛生物能源与过程研究所联合培养硕士生. 主要从 事锂硫电池电解质的研究.

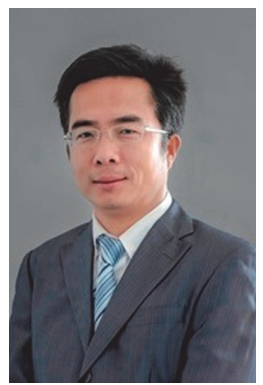

崔光磊, 研究员, 博士生导师, 2005 年于中国科学院化学 所获得有机化学博士学位, 2005 年 9 月至 2009 年 2 月先后在 德国马普协会高分子所和固态所从事博士后研究. 2009 年 2 月 起于中科院青岛生物能源与过程所工作. 2009 年入选中国科 学院 “百人计划” (终期评估优秀), 2009 年获山东省自然科学 杰出青年基金资助, 2015 年入选山东省 “泰山学者特聘专家”, 2016 年获国家自然科学杰出青年基金资助. 主要从事低成本 高效能源储存与转换器件的研究, 作为负责人和主要参与者 承担国家自然科学杰出青年基金, 国家 973 计划, 863 计划, 国 家自然科学基金面上项目, 省部级及中科院先导专项, 企业 横向项目等多项科研项目.

\section{References}

[1] Etacheri, V.; Marom, R.; Elazari, R.; Salitra, G.; Aurbach, D. Energy Environ. Sci. 2011, 4, 3243.

[2] Goodenough, J. B.; Kim, Y. Chem. Mater. 2010, 22, 587.

[3] Goodenough, J. B.; Park, K.-S. J. Am. Chem. Soc. 2013, 135, 1167.

[4] Xu, K. Chem. Rev. 2004, 104, 4303.

[5] He, Q.; Zhang, C.; Li, X.; Wang, X.; Mu, P.; Jiang, J. X. Acta Chim. Sinica 2018, 76, 202 (in Chinese). (贺倩, 张崇, 李晓, 王雪, 牟攀, 蒋加兴, 化学学报, 2018, 76, 202.)

[6] Wang, C. Q.; Qiu, F. L.; Deng, H.; Zhang, X. Y.; He, P.; Zhou, H. S. Acta Chim. Sinica 2017, 75, 241 (in Chinese). (王超强, 邱飞龙, 邓 瀚, 张晓禹, 何平, 周豪慎, 化学学报, 2017, 75, 241.)

[7] Gu, X. Y.; Hong, Y.; Ai, G.; Wang, C. Y.; Mao, W. F. Acta Chim. Sinica 2018, 76, 644 (in Chinese). (顾晓瑜, 洪晔, 艾果, 王朝阳, 毛文峰, 化学学报, 2018, 76, 644.)

[8] Kalhoff, J.; Eshetu, G. G.; Bresser, D.; Passerini, S. ChemSusChem 2015, 8, 2765.

[9] Lahiri, A.; Shah, N.; Dales, C. IEEE Spectrum 2018, 55, 34.

[10] Li, J.; Tang, S.; Huang, J.; Tao, X.; Zhou, Y.; Li, D. New Chem. Mater. 2012, 40, 6 (in Chinese). (李军, 唐盛贺, 黄际伟, 陶熏, 周燕, 李大光, 化工新型材料, 2012, 40, 6.)

[11] Liu, X.; Yu, L. Battery 2004, 34, 449.

[12] Gao, J.; Lowe, M. A.; Kiya, Y.; Abruña, H. D. J. Phys. Chem. C 2011, 115, 25132.

[13] Ahmad, S. Ionics 2009, 15, 309.

[14] Chen, R.; Qu, W.; Guo, X.; Li, L.; Wu, F. Mater. Horiz. 2016, 3, 487.

[15] Azov, V. A.; Egorova, K. S.; Seitkalieva, M. M.; Kashina, A. S.;
Ananikov, V. P. Chem. Soc. Rev. 2018, 47, 1250.

[16] Borodin, O.; Vatamanu, J.; Ren, X. J. Am. Chem. Soc. 2018, 256, 1155 .

[17] Messaggi, F.; Ruggeri, I.; Genovese, D.; Zaccheroni, N.; Arbizzani, C.; Soavi, F. Electrochim. Acta 2017, 245, 288.

[18] Suo, L.; Fang, Z.; Hu, Y.-S.; Chen, L. Chinese Phys. B 2016, 25, 21.

[19] Suo, L.; Hu, Y.-S.; Li, H.; Armand, M.; Chen, L. Nat. Commun. 2013, 4, 1481.

[20] Zeng, Z.; Murugesan, V.; Han, K. S.; Jiang, X.; Cao, Y.; Xiao, L.; Ai, X.; Yang, H.; Zhang, J.-G.; Sushko, M. L.; Liu, J. Nature Energy 2018, 3, 674 .

[21] Cao, R.; Mishra, K.; Li, X.; Qian, J.; Engelhard, M. H.; Bowden, M. E.; Han, K. S.; Mueller, K. T.; Henderson, W. A.; Zhang, J.-G. Nano Energy 2016, 30, 825.

[22] Suo, L.; Borodin, O.; Gao, T.; Olguin, M.; Ho, J.; Fan, X.; Luo, C.; Wang, C.; Xu, K. Science 2015, 350, 938.

[23] McOwen, D. W.; Seo, D. M.; Borodin, O.; Vatamanu, J.; Boyle, P. D.; Henderson, W. A. Energy Environ. Sci. 2014, 7, 416.

[24] Wang, J.; Yamada, Y.; Sodeyama, K.; Chiang, C. H.; Tateyama, Y.; Yamada, A. Nat. Commun. 2016, 7, 12032.

[25] Fan, X. L.; Ji, X.; Han, F. D.; Yue, J.; Chen, J.; Chen, L.; Deng, T.; Jiang, J. J.; Wang, C. S. Sci. Adv. 2018, 4, 10.

[26] Peng, Z.; Zhao, N.; Zhang, Z.; Wan, H.; Lin, H.; Liu, M.; Shen, C.; He, H.; Guo, X.; Zhang, J.-G.; Wang, D. Nano Energy 2017, 39, 662.

[27] Wang, L. L.; Ma, J.; Wang, C.; Yu, X. R.; Liu, R.; Jiang, F.; Sun, X. W.; Du, A. B.; Zhou, X. H.; Cui, G. L. Adv. Sci. 2019, 6, 11.

[28] Yoo, E.; Zhou, H. ACS Appl. Mater. Interfaces 2017, 9, 21307.

[29] Deng, B. W.; Sun, D. M.; Wan, Q.; Wang, H.; Chen, T.; Li, X.; Qu, M. Z.; Peng, G. C. Acta Chim. Sinica 2018, 76, 259 (in Chinese). (邓 邦为, 孙大明, 万琦, 王昊, 陈滔, 李璇, 篗美臻, 彭工厂, 化学 学报, 2018, 76, 259.)

[30] Yamada, Y.; Wang, J.; Ko, S.; Watanabe, E.; Yamada, A. Nature Energy 2019, 4, 269.

[31] Yamada, Y.; Yamada, A. J. Electrochem. Soc. 2015, 162, A2406.

[32] Chen, S.; Zheng, J.; Yu, L.; Ren, X.; Engelhard, M. H.; Niu, C.; Lee, H.; Xu, W.; Xiao, J.; Liu, J.; Zhang, J.-G. Joule 2018, 2, 1548.

[33] Zheng, J.; Chen, S.; Zhao, W.; Song, J.; Engelhard, M. H.; Zhang, J.-G. ACS Energy Lett. 2018, 3, 315.

[34] Xia, L.; Yu, L. P.; Hu, D.; George, C. Z. Acta Chim. Sinica 2017, 75, 1183 (in Chinese). (夏兰, 余林颇, 胡笛, 陈政, 化学学报, 2017, 75, 1183.)

[35] Dagger, T.; Meier, V.; Hildebrand, S.; Brueggemann, D.; Winter, M.; Schappacher, F. M. Energy Technol. 2018, 6, 2001.

[36] Wang, J.; Lin, F.; Jia, H.; Yang, J.; Monroe, C. W.; NuLi, Y. Angew. Chem. Int. Ed. 2014, 53, 10099.

[37] Wang, Q.; Jiang, L.; Yu, Y.; Sun, J. Nano Energy 2019, 55, 93.

[38] Yang, H.; Guo, C.; Chen, J.; Naveed, A.; Yang, J.; Nuli, Y.; Wang, J. Angew. Chem. Int. Ed. 2019, 58, 791.

[39] Yang, H.; Li, Q.; Guo, C.; Naveed, A.; Yang, J.; Nuli, Y.; Wang, J. Chem Commun (Camb) 2018, 54, 4132.

[40] Ye, T.; Li, D.; Liu, H.; She, X.; Xia, Y.; Zhang, S.; Zhang, H.; Yang, D. Macromolecules 2018, 51, 9360.

[41] Zheng, J.; Ji, G.; Fan, X.; Chen, J.; Li, Q.; Wang, H.; Yang, Y.; DeMella, K. C.; Raghavan, S. R.; Wang, C. Adv. Energy Mater. 2019, 9, 1803774.

[42] Jia, H.; Zou, L.; Gao, P.; Cao, X.; Zhao, W.; He, Y.; Engelhard, M. H.; Burton, S. D.; Wang, H.; Ren, X.; Li, Q.; Yi, R.; Zhang, X.; Wang, C.; Xu, Z.; Li, X.; Zhang, J.-G.; Xu, W. Adv. Energy Mater. 2019, 9, 1900784.

[43] Du, J.; Lin, N.; Qian, Y. T. Acta Chim. Sinica 2017, 75, 147 (in Chinese). (杜进, 林宁, 钱逸泰, 化学学报, 2017, 75, 147.)

[44] Chen, S.; Zheng, J.; Mei, D.; Han, K. S.; Engelhard, M. H.; Zhao, W.; Xu, W.; Liu, J.; Zhang, J. G. Adv. Mater. 2018, 30, 1706102.

[45] Ren, X.; Chen, S.; Lee, H.; Mei, D.; Engelhard, M. H.; Burton, S. D.; Zhao, W.; Zheng, J.; Li, Q.; Ding, M. S.; Schroeder, M.; Alvarado, J.; Xu, K.; Meng, Y. S.; Liu, J.; Zhang, J.-G.; Xu, W. Chem 2018, 4, 1877.

[46] Ma, G.; Wang, L.; He, X.; Zhang, J.; Chen, H.; Xu, W.; Ding, Y. Adv. Energy Mater. 2018, 1, 5446.

[47] Ren, X.; Zou, L.; Cao, X.; Engelhard, M. H.; Liu, W.; Burton, S. D.; Lee, H.; Niu, C.; Matthews, B. E.; Zhu, Z.; Wang, C.; Arey, B. W.; Xiao, J.; Liu, J.; Zhang, J.-G.; Xu, W. Joule 2019, 3, 1.

[48] Dong, X.; Lin, Y.; Li, P.; Ma, Y.; Huang, J.; Bin, D.; Wang, Y.; Qi, Y.; Xia, Y. Angew. Chem. Int. Ed. 2019, 58, 5623.

[49] Huang, J.; Sun, Y.; Wang, Y.; Zhang, Q. Acta Chim. Sinica 2017, 75, 173 (in Chinese). (黄佳琦, 孙漟智, 王云飞, 张强, 化学学报, 
2017, 75, 173.)

[50] Chen, K. F.; Xue, D. F. Chin. J. Chem. 2017, 35, 861.

[51] Li, W. F.; Ma, Q.; Zheng, Z. Z.; Zhang, Y. G. Acta Chim. Sinica 2017, 75, 225 (in Chinese). (李宛飞, 马倩, 郑召召, 张跃钢, 化学 学报, 2017, 75, 225.)

[52] He, F.; Wu, X.; Qian, J.; Cao, Y.; Yang, H.; Ai, X.; Xia, D. J. Mater. Chem. A 2018, 6, 23396.

[53] Manthiram, A.; Fu, Y.; Chung, S.-H.; Zu, C.; Su, Y.-S. Chem. Rev. 2014, 114, 11751.

[54] Manthiram, A.; Fu, Y.; Su, Y.-S. Acc. Chem. Res. 2013, 46, 1125.

[55] Conder, J.; Bouchet, R.; Trabesinger, S.; Marino, C.; Gubler, L.; Villevieille, C. Nature Energy 2017, 2, 17069.

[56] Li, X.; Banis, M.; Lushington, A.; Yang, X.; Sun, Q.; Zhao, Y.; Liu, C.; Li, Q.; Wang, B.; Xiao, W.; Wang, C.; Li, M.; Liang, J.; Li, R.; $\mathrm{Hu}$, Y.; Goncharova, L.; Zhang, H.; Sham, T. K.; Sun, X. Nat. Commun. 2018, 9, 4509

[57] Mikhaylik, Y. V.; Akridge, J. R. J. Electrochem. Soc. 2004, 151, A1969.

[58] Cuisinier, M.; Cabelguen, P. E.; Adams, B. D.; Garsuch, A.; Balasubramanian, M.; Nazar, L. F. Energy Environ. Sci. 2014, 7, 2967.

[59] Moon, H.; Mandai, T.; Tatara, R.; Ueno, K.; Yamazaki, A.; Yoshida, K.; Seki, S.; Dokko, K.; Watanabe, M. J. Phys. Chem. C 2015, 119, 3957.

[60] Jiang, J.; Gao, D.-S.; Li, Z.-H.; Su, G.-Y.; Wang, C.-W.; Liu, L.; Ding, Y.-H. Chem. J. Chin. Univ. 2006, 27, 1319 (in Chinese). (蒋 晶, 高德淑, 李朝晖, 苏光耀, 王承位, 刘黎, 丁燕怀, 高等学校 化学学报, 2006, 27, 1319.)
[61] Jing, J.; Guangyao, S. U. Battery 2005, 35, 474.

[62] Taige, M. A.; Hilbert, D.; Schubert, T. J. S. Zeitschrift für Physikalische Chemie 2012, 226, 129.

[63] Lewandowski, A.; Swiderska-Mocek, A. J. Power Sources 2009, $194,601$.

[64] Qiu, H.; Zhao, J.; Zhou, X.; Cui, G. Acta Chim. Sinica 2018, 76, 749 (in Chinese). (邱华玉, 赵井文, 周新红, 崔光否, 化学学报, 2018 , 76, 749.)

[65] Dokko, K.; Tachikawa, N.; Yamauchi, K.; Tsuchiya, M.; Yamazaki, A.; Takashima, E.; Park, J.-W.; Ueno, K.; Seki, S.; Serizawa, N.; Watanabe, M. J. Electrochem. Soc. 2013, 160, A1304.

[66] Ueno, K.; Murai, J.; Ikeda, K.; Tsuzuki, S.; Tsuchiya, M.; Tatara, R. Mandai, T.; Umebayashi, Y.; Dokko, K.; Watanabe, M. J. Phys. Chem. C 2015, 120, 15792.

[67] Kim, S.-W.; Seo, D.-H.; Ma, X.; Ceder, G.; Kang, K. Adv. Energy Mater. 2012, 2, 710.

[68] Slater, M. D.; Kim, D.; Lee, E.; Johnson, C. S. Adv. Funct. Mater. 2013, 23, 947.

[69] Xiang, X.; Lu, Y.; Chen, J. Acta Chim. Sinica 2017, 75, 154 (in Chinese). (向兴德, 卢艳莹, 陈军, 化学学报, 2017, 75, 154.)

[70] Zou, W.; Fan, C.; Li, J. Z. Chin. J. Chem. 2017, 35, 79

[71] Qu, L. P.; Ren, T.; Wang, N.; Shi, Y. L.; Zhuang, Q. C. Acta Chim. Sinica 2019, 77, 634 (in Chinese). (渠璐平，任肜，王宁，史月丽， 庄全超, 化学学报, 2019, 77, 634.)

[72] Doi, T.; Shimizu, Y.; Hashinokuchi, M.; Inaba, M. J. Electrochem Soc. 2017, 164, A6412.

(Cheng, B.) 